\title{
CHARACTERIZATION OF SOME MATRIX CLASSES INVOLVING PARANORMED SEQUENCE SPACES
}

\author{
BINOD CHANDRA TRIPATHY AND MAUSUMI SEN
}

\begin{abstract}
In this article we characterize some matrix classes with one member as $m(p)$ or $m_{0}(p)$ or $c(p)$ or $c_{0}(p)$. Some of these results generalize the existing results. Some are new proved in the general setting.
\end{abstract}

\section{Introduction}

Throughout the article $w, \gamma, \gamma_{0}, c, c_{0}, \ell_{\infty}$ denote the spaces of all, summable, summable to zero, convergent, null and bounded sequences respectively. The notion of statistical convergence of sequences was introduced by Fast [3], Schoenberg [12] and Buck [1] independently. Later on the idea was exploited from sequence space point of view and linked with summability by Fridy [4], Šalát [11], Kolk [5], Rath and Tripathy [10], Connor [2], Tripathy([14], [15]) and many others. The basic idea depends on the density of the subsets of $N$, the set of natural numbers. A subset $E$ of $N$ is said to have density $\delta(E)$ if $\delta(E)=\lim _{n \rightarrow \infty} \frac{1}{n} \sum_{k=1}^{n} \chi_{E}(k)$ exists, where $\chi_{E}$ is the characteristic function of $E$.

A sequence $\left(x_{k}\right)$ is said to be statistically convergent to $L$ if for every $\varepsilon<0, \delta(\{k \in$ $\left.\left.N:\left|x_{k}-L\right| \geq \varepsilon\right\}\right)=0$. We write $x_{k} \stackrel{\text { stat }}{\longrightarrow} L$ or stat-lim $x_{k}=L$.

Tripathy and Sen [17] have generalized the notion on extending it for paranormed sequence spaces. The notion of paranormed sequence space was first studied by Nakano [9] and Simons [13]. Later on it was exploited by Maddox [8], Lascarides and Maddox [7], Lascarides [6], Tripathy [16] and many others. Throughout $p=\left(p_{k}\right) \in \ell_{\infty}$ denote a non-negative sequence of real numbers. We write $r_{k}=\frac{1}{p_{k}}$ for all $k \in N$.

The following known paranormed sequence spaces will be used.

$$
\begin{aligned}
c(p) & =\left\{\left(x_{k}\right) \in w:\left|x_{k}-L\right|^{p_{k}} \rightarrow 0, \text { as } k \rightarrow \infty \text { for some } L\right\} \\
c_{0}(p) & =\left\{\left(x_{k}\right) \in w:\left|x_{k}\right|^{p_{k}} \rightarrow 0, \text { as } k \rightarrow \infty\right\} \\
\ell_{\infty}(p) & =\left\{\left(x_{k}\right) \in w: \sup _{k}\left|x_{k}\right|^{p_{k}}<\infty\right\} \\
\bar{c}(p) & =\left\{\left(x_{k}\right) \in w:\left|x_{k}-L\right|^{p_{k}} \stackrel{\text { stat }}{\longrightarrow} 0, \text { for some } L\right\} \\
\bar{c}_{0}(p) & =\left\{\left(x_{k}\right) \in w:\left|x_{k}\right|^{p_{k}} \stackrel{\text { stat }}{\longrightarrow} 0\right\} .
\end{aligned}
$$

Received and revised August 23, 2004.

2000 Mathematics Subject Classification. Primary 40C05; Secondary 40A05.

Key words and phrases. Density, paranormed sequence space, statistical convergence. 
We write $m(p)=\bar{c}(p) \cap \ell_{\infty}(p)$ and $m_{0}(p)=\bar{c}_{0}(p) \cap \ell_{\infty}(p)$.

The above spaces are paranormed by $g\left(\left(x_{k}\right)\right)=\sup _{k}\left|x_{k}\right|^{\frac{p_{k}}{M}}$, where $M=\max \left(1, \sup p_{k}\right)$.

\section{Preliminaries}

The following results will be used for establishing the results of this article.

Lemma 1. (Tripathy and Sen [17], Theorem 2). The space $m(p)$ is a closed subspace of $\ell_{\infty}(p)$.

Lemma 2. (Lascarides [6], Remark, P.494). Let $p, q \in \ell_{\infty}$. Then we have $A=$ $\left(a_{n k}\right) \in\left(c_{0}(p), \ell_{\infty}(q)\right)$ if and only if there exists an absolute constant $D>1$ such that

$$
\sup _{n}\left\{\sum_{k}\left|a_{n k}\right| D^{-r_{k}}\right\}^{q_{n}}<\infty
$$

In view of the above lemma and using standard techniques we have the following result.

Lemma 3. Let $\left(p_{k}\right) \in \ell_{\infty}$. Then $A=\left(a_{n k}\right) \in\left(\ell_{\infty}, \ell_{\infty}(p)\right)$ if and only if

$$
\sup _{n}\left\{\sum_{k}\left|a_{n k}\right|\right\}^{q_{n}}<\infty
$$

Lemma 4. (Lascarides [6], Theorem 9.) Let $p \in \ell_{\infty}(p)$. Then $A=\left(a_{n k}\right) \in(c(p), c)$ if and only if there exists an absolute constant $D>1$ such that

$$
\begin{aligned}
& \sup _{n} \sum_{k}\left|a_{n k}\right| D^{-r_{k}}<\infty, \\
& \lim _{n \rightarrow \infty} a_{n k}=\alpha_{k} \text { exists for every fixed } k . \\
& \lim _{n \rightarrow \infty} \sum_{k} a_{n k}=\alpha_{n k} \text { exists. }
\end{aligned}
$$

Lemma 5. Let $0<\inf p_{k} \leq \sup p_{k}<\infty$. Then for any linear subspace $X$ of $\ell_{\infty}(p)$, the following are equivalent:

$X$ is complete with respect to $g$.

If $\sum_{k} a_{n k}$ converges uniformly to $a_{n}$ for each $n \in N$ and for each $k \in N$,

$a^{k}=\left(a_{n k}\right)_{n \in N} \in X$, then $a=\left(a_{n}\right) \in X$. 
Proof. $(2.6) \Rightarrow(2.7)$. Suppose $\sum_{k} a_{n k}$ converges uniformly to $a_{n}$ for each $n \in N$ and $a^{k}=\left(a_{n k}\right)_{n \in N} \in X$ for each $k \in N$. Since $X$ is linear, so $s^{j}=\sum_{k=1}^{j} a^{k} \in X, j \in N$. We have $\left\|s^{j}-a\right\|=\sup _{n}\left|\sum_{k>j} a_{n k}\right|^{\frac{p_{k}}{M}}$.

Since the convergence of $\sum_{k} a_{n k}$ is uniform, so given $1>\varepsilon>0$, there exists $j_{0}$ such that $\left\|s^{j}-a\right\|<\varepsilon^{\frac{h}{M}}$ for all $j>j_{0}$.

Thus we have $a \in X$, since $X$ is complete.

$(2.7) \Rightarrow(2.6)$. Let $\left(x^{m}\right)$, where $x^{m}=\left(x_{k}^{m}\right)_{k \in N}$, be a Cauchy sequence in $X$. Then $\left(x^{m}\right)$ converges (say to $x$ ) in $\ell_{\infty}(p)$, since $\ell_{\infty}(p)$ is complete. Write $a_{k m}=x_{k}^{m}-x_{k}^{m-1}$ $\left(x_{k}^{0}=0\right)$. Then $\sum_{m} a_{k m}$ converges uniformly to $x_{k}$ and $\left(a_{k m}\right)_{k \in N}=a^{m} \in X$.

Note 1. Taking $p_{n}=1$ for all $n \in N$, one will get Lemma 4 of Rath and Tripathy [10] as particular case.

Lemma 6. Let $\left(p_{k}\right) \in \ell_{\infty}$, then $A=\left(a_{n k}\right) \in\left(\gamma, \ell_{\infty}(p)\right)$ if and only if

$$
\begin{aligned}
& \quad T=\sup _{n}\left\{\sum_{k}\left|\Delta a_{n k}\right|\right\}^{p_{n}}<\infty \text {, where } \Delta a_{n k}=a_{n k}-a_{n, k+1} \text {, for all } k \in N, \\
& \text { and } \\
& \left(a_{m}\right) \in \ell_{\infty}(p) .
\end{aligned}
$$

Proof. Let $s=\left(s_{k}\right) \in \gamma$ and $S_{n}=\sum_{k=1}^{n} s_{k} \rightarrow S$ as $n \rightarrow \infty$. Then by Abel's summation formula we have

$$
A_{n} s=\sum_{k=1}^{\infty} a_{n k} s_{k}=S a_{n 1}+\sum_{k=1}^{\infty} \Delta a_{n k}\left(S_{k}-S\right)
$$

The rest of the proof is a routine work in view of Lemma 2 and using standard techniques.

The proof of the following result is a routine work in view of Lemma 6 .

Lemma 7. Let $\left(p_{k}\right) \in \ell_{\infty}$, then $A=\left(a_{n k}\right) \in\left(\gamma_{0}, \ell_{\infty}(p)\right)$ if and only if (2.8) holds.

\section{The Main Results}

In this section we establish the results of this article.

Theorem 1. Let $0<\inf p_{k} \leq \sup p_{k}<\infty$. Then $A=\left(a_{n k}\right) \in(\gamma, m(p))$ if and only if (2.8) holds and

$$
\left(a_{n k}\right)_{n \in N} \in m(p), \text { for every } k \in N \text {. }
$$


Proof. The necessity of (2.8) follows from the inclusion $(\gamma, m(p)) \subset\left(\gamma, \ell_{\infty}(p)\right)$ and Lemma 5 and that of $(3.1)$ on considering the sequence $e_{k}=(0,0, \cdots 0,1,0, \cdots)$ in $\gamma$ where the only 1 appears at the $k$-th place.

Sufficiency. Let $s=\left(s_{k}\right) \in \gamma$. We have by (3.1) that $\left(\Delta a_{n k}\right)_{n \in N} \in m(p)$ for all $k=1,2,3, \cdots$.

Hence we have $S a_{n 1}+\sum_{k \leq j_{0}} \Delta a_{n k}\left(S_{k}-S\right) \in m(p)$, by the linearity.

Next we have

$$
\begin{aligned}
\left|\sum_{k>j_{0}} \Delta a_{n k}\left(S_{k}-S\right)\right| & \leq T^{\frac{1}{h}} \max _{k>j_{0}}\left|S_{k}-S\right| \\
& \rightarrow 0, \text { uniformly in } n \text { as } j_{0} \rightarrow \infty
\end{aligned}
$$

Hence by Lemma 1, Lemma 5 and (2.8) we have $A s \in m(p)$.

This completes the proof of the theorem.

The proof of the following result is obvious in view of the above result.

Corollary 1. Let $0<\inf p_{k} \leq \sup p_{k}<\infty$. Then $A=\left(a_{n k}\right) \in\left(\gamma_{0}, m(p)\right)$ if and only if (2.8) holds and $\left(a_{n k}\right)_{n \in N} \in m_{0}(p)$, for every $k \in N$.

Following the techniques of Tripathy [15] and the arguments of Theorem 1, we have the following result.

Theorem 2. Let $0<\inf p_{k} \leq \sup p_{k}<\infty$. Then $A=\left(a_{n k}\right) \in(\gamma, m(p) ; P)$ if and only if $(2.8)$ holds and $\left(a_{n k}-1\right)_{n \in N} \in m_{0}(p)$, for all $k=1,2,3, \cdots$. In this transformation, the limit is preserved.

Theorem 3. Let $0<\inf p_{k} \leq \sup p_{k}<\infty$. Then $A=\left(a_{n k}\right) \in\left(\gamma_{0}, m(p)\right)$ if and only if (2.8) holds and

$$
\left(\Delta a_{n k}\right)_{n \in N} \in m(p), \text { for each fixed } k \in N \text {. }
$$

Proof. The necessity of (2.8) follows from the inclusion $\left(\gamma_{0}, m(p)\right) \subset\left(\gamma_{0}, \ell_{\infty}(p)\right)$ and Lemma 7 . The necessity of (3.2) follows on considering the series $\left(s_{k}\right)$ whose $k$-th term is 1 and $(k-1)$-th term is -1 and rest are zero.

Putting $S=0$ in $(2.10)$ we have

$$
A_{n} s=\sum_{k=1}^{\infty} \Delta a_{n k} S_{k}, \text { for all } n=1,2,3, \cdots
$$

Following the techniques of Theorem 1, it can be shown that $A s \in m(p)$. This completes the proof of the theorem.

The following result is an easy consequence of the above theorem. 
Corollary 2. Let $0<\inf p_{k} \leq \sup p_{k}<\infty$. Then $A=\left(a_{n k}\right) \in\left(\gamma_{0}, m(p)\right)$ if and only if (2.8) holds and

$$
\left(\Delta a_{n k}\right)_{n \in N} \in m_{0}(p), \text { for all } k=2,3,4, \cdots .
$$

Note 2. Taking $p_{n}=1$ for all $n \in N$ in Theorem 1, Corollary 1, Theorem 2, Theorem 3 , and Corollary 2, we have the characterization of the matrix classes $(\gamma, m),\left(\gamma, m_{0}\right)$, $(\gamma, m ; P),\left(\gamma_{0}, m\right)$ and $\left(\gamma_{0}, m_{0}\right)$ i.e. the results of Tripathy [15] as particular cases.

It is well known that $c(p) \subset \ell_{\infty}$ if $p \in \ell_{\infty}$. The proofs of the following results are routine works in view of Lemma 5 , taking $p_{n}=1$ for all $n \in N$ and the technique for establishing the above results.

Proposition 4. Let $p \in \ell_{\infty}$ and $X$ be either $c(p)$ or $c_{0}(p)$. Then

$$
\begin{aligned}
A= & \left(a_{n k}\right) \in(\gamma, X) \text { if and only if }(2.8) \text { holds with } p_{n}=1 \text { for all } n \in N \\
& \text { and }\left(a_{n k}\right)_{n \in N} \in X \text { for all } k \in N . \\
A= & \left(a_{n k}\right) \in\left(\gamma_{0}, X\right) \text { if and only if }(2.8) \text { holds with } p_{n}=1 \text { for all } n \in N \\
& \text { and }\left(\Delta a_{n k}\right)_{n \in N} \in X \text { for all } k \in N .
\end{aligned}
$$

Proposition 5. Let $p \in \ell_{\infty}$. Then $A=\left(a_{n k}\right) \in(\gamma, c(p) ; P)$ if and only if (2.8) holds and

$$
\left|a_{n k}-1\right|^{p_{n}} \rightarrow 0, \text { as } n \rightarrow \infty \text { for all } k \in N \text {. }
$$

Theorem 6. Let $0<\inf p_{k} \leq \sup p_{k}<\infty$. Then $A=\left(a_{n k}\right) \in(m(p), c)$ if and only if (2.3), (2.4), (2.5) hold and

$$
\lim _{n \rightarrow \infty} \sum_{n \in S}\left|a_{n k}-\alpha_{k}\right| F^{r_{k}}=0 \text { for each } S \subset N \text { with } \delta(S)=0 \text { and for all } F>1 .
$$

Proof. The necessity of $(2.3)$ is clear in view of the inclusion $(m(p), c) \subset(c(p), c)$. The necessity of (2.4) and (2.5) follow on considering the sequences $e_{k}$ and $e=(1,1,1, \cdots)$ respectively.

Next suppose $A \in(m(p), c)$ but $\lim _{n \rightarrow \infty} \sum_{k \in S}\left|a_{n k}-\alpha_{k}\right| F^{r_{k}} \neq 0$ for some $F>1$. Let us define the matrix $B=\left(b_{n k}\right)$ as follows:

$$
b_{n k}= \begin{cases}\left(a_{n k}-\alpha_{k}\right) F^{r_{k}}, & k \in S \\ 0, & \text { othewise }\end{cases}
$$

for all $n \in N$.

Then

$$
\lim _{n \rightarrow \infty} \sum_{k=1}^{\infty}\left|b_{n k}\right|=\lim _{n \rightarrow \infty} \sum_{k \in S}\left|a_{n k}-\alpha_{k}\right| F^{r_{k}} \neq 0
$$


Thus $B \notin\left(\ell_{\infty}, c\right)$, so there exists $x=\left(x_{k}\right) \in \ell_{\infty}$ with $\sup _{k}\left|x_{k}\right|=1$ such that $B x \notin c i . e$.

$$
\left(\sum_{k \in S}\left(a_{n k}-\alpha_{k}\right) F^{r_{k}} x_{k}\right) \notin c .
$$

Define the sequence $y=\left(y_{k}\right)$ as follows:

$$
y_{k}= \begin{cases}x_{k} F^{r_{k}}, & k \in S \\ 0, & \text { othewise }\end{cases}
$$

Then $y \in m(p)$. We have for each $n \in N$,

$$
A_{n} y=\sum_{k=1}^{\infty} a_{n k} y_{k}=\sum_{k \in S} a_{n k} x_{k} F^{r_{k}}=\sum_{k \in S}\left(a_{n k}-\alpha_{k}\right) x_{k} F^{r_{k}}+\sum_{k \in S} \alpha_{k} x_{k} F^{r_{k}} .
$$

Thus $\left(A_{n} y\right) \notin c$ by (3.7). Hence the necessity of (3.6) follows.

Sufficiency. Let $x=\left(x_{k}\right) \in m(p)$. Then there exists $y=\left(y_{k}\right) \in c(p)$ and $z=\left(z_{k}\right) \in$ $\delta_{0}(p)$ such that $x_{k}=y_{k}+z_{k}$ for all $k \in N$. By $(2.3),(2.4),(2.5)$ we have $A \in(c(p), c)$. Thus $A y=\left(A_{n} y\right) \in c$ whenever $y \in c(p)$. Next we have

$$
\begin{aligned}
\left|\sum_{k=1}^{\infty} a_{n k} z_{k}-\sum_{k=1}^{\infty} \alpha_{k} z_{k}\right| & =\left|\sum_{k \in S}\left(a_{n k}-\alpha_{k}\right) z_{k}\right| \\
& \leq \sum_{k \in S}\left|a_{n k}-\alpha_{k}\right| F^{r_{k}} \rightarrow 0, \text { as } n \rightarrow \infty .
\end{aligned}
$$

Therefore $A z \in c$. Hence $A \in(m(p), c)$. This completes the proof of the theorem.

The proof of the following two results is a routine work in view of the proof of the above result.

Corollary 3. $A=\left(a_{n k}\right) \in(m(p), c ; P)$ if and only if the conditions (2.3), (2.4), (2.5) with $\alpha=1$ and (3.6) with $\alpha_{k}=0$ for each $k=1,2,3, \ldots$ hold.

Corollary 4. $A=\left(a_{n k}\right) \in\left(m_{0}(p), c\right)$ if and only if (2.3), (2.4) and (3.6) hold.

Theorem 7. Let $p, q \in \ell_{\infty}$, then $A=\left(a_{n k}\right) \in\left(m_{o}(p), \ell_{\infty}(q)\right)$ if and only if $(2.1)$ holds and

$$
\sup _{n}\left\{\sum_{k}\left|a_{n k}\right| F^{r_{k}}\right\}^{q_{n}}<\infty \text { for each } S \subset N \text { with } \delta(S)=0 \text { and for all } F>1 \text {. }
$$

Proof. The necessity of (2.1) follows from the inclusion $\left(m_{0}(p), \ell_{\infty}(q)\right) \subset\left(c_{0}(p)\right.$, $\left.\ell_{\infty}(q)\right)$. Next let $S \subset N$ be such that $\delta(S)=0$ and $\sup \left\{\sum_{k}\left|a_{n k}\right| F^{r_{k}}\right\}^{q_{n}}=\infty$ for some $F>1$. Define a matrix $B=\left(b_{n k}\right)$ as follows:

$$
b_{n k}= \begin{cases}a_{n k} F^{r_{k}}, & k \in S \\ 0, & \text { othewise }\end{cases}
$$


for all $n \in N$.

We have

$$
\sup _{n}\left\{\sum_{k=1}^{\infty}\left|b_{n k}\right|\right\}^{q_{n}}=\sup _{n}\left\{\sum_{k \in S}\left|a_{n k}\right| F^{r_{k}}\right\}^{q_{n}}=\infty .
$$

Hence $B \notin\left(\ell_{\infty}, \ell_{\infty}(q)\right)$. Thus there exists $x=\left(x_{k}\right) \in \ell_{\infty}$ with $\sup _{n}\left|x_{k}\right|=1$ such that

$$
\left(\sum_{k=1}^{\infty} b_{n k} x_{k}\right)=\left(\sum_{k \in S} a_{n k} F^{r_{k}} x_{k}\right) \notin \ell_{\infty}(q)
$$

Define the sequence $y=\left(y_{k}\right)$ as follows:

$$
y_{k}= \begin{cases}x_{k} F^{r_{k}}, & k \in S \\ 0, & \text { othewise. }\end{cases}
$$

Then clearly $\left(y_{k}\right) \in m_{0}(p)$. But $\left(A_{n} y\right) \notin \ell_{\infty}(q)$ by $(3.9)$, as such we arrive at a contradiction. Thus the necessity of (3.8) follows.

Sufficiency. Let $x=\left(x_{k}\right) \in m_{0}(p)$. Then for a given $0<\varepsilon<1, \delta(K)=\delta(\{k \in N$ : $\left.\left.\left|x_{k}\right|^{p_{k}}<\varepsilon\right\}\right)=1$ and $\left|x_{k}\right|^{p_{k}}<F$ for all $k \in N$. Let $D=\varepsilon^{-1}$, then $D>1$. If $k \in K$, then $\left|x_{k}\right|<D^{-r_{k}}$ and for $k \notin K$, we have $\left|x_{k}\right|<F^{r_{k}}$. We have

$$
\begin{aligned}
\left|\sum_{k=1}^{\infty} a_{n k} x_{k}\right|^{q_{n}} & \leq C\left[\left\{\sum_{k \in K}\left|a_{n k} \| x_{k}\right|\right\}^{q_{n}}+\left\{\sum_{k \in K^{c}}\left|a_{n k} \| x_{k}\right|\right\}^{q_{n}}\right] \\
& \leq C\left[\left\{\sum_{k \in K}\left|a_{n k}\right| D^{-r_{k}}\right\}^{q_{n}}+\left\{\sum_{k \in K^{c}}\left|a_{n k}\right| F^{r_{k}}\right\}^{q_{n}}\right] \\
& <\infty .
\end{aligned}
$$

Thus $A \in\left(m_{0}(p), \ell_{\infty}(q)\right)$. This completes the proof of the theorem.

\section{References}

[1] R. C. Buck, Generalized asymptotic density, Amer. Jour. Math. 75(1953), 335-346.

[2] J. S. Connor, The statistical and strong p-Cesàro convergence of sequences, Analysis 8(1988), 47-63.

[3] H. Fast, Sur la convergence statistique, Colloq. Math. 2(1951), 241-244.

[4] J. A. Fridy, Statistical limit points, Porc. Amer. Math. Sos. 118(1993), 1187-1192.

[5] E. Kolk, Matrix summability of statistically convergent sequences, Analysis 13(1993), 77-83.

[6] C. G. Lascarides, A study of certain sequence spaces of Maddox and a generalization of a theorem of Iyer, Pacific. Jour. Math. 38(1971), 487-500.

[7] C. G. Lascarides and I. J. Maddox, Matrix transformation between some classes of sequences, Proc. Camb. Phil. Soc. 68(1970), 99-104.

[8] I. J. Maddox, Paranormed sequence spaces generated by infinite matrices, Proc. Camb. Phil. Soc. 64(1968), 335-340. 
[9] H. Nakano, Modulared sequence space, Proc. Japan Acad. 27(1951), 508-512.

[10] D. Rath and B. C. Tripathy, Matrix maps on sequence spaces associated with sets of integers, Ind. Jour. Pure \& Appl. Math. 27(1996), 197-206.

[11] T. Šalát, On statistically convergent sequences of real numbers, Math. Slovaca 30(1980), 139-150.

[12] I. J. Schoenberg, The integrability of certain functions and related summability methods, Amer. Math. Monthly 66(1959), 361-375.

[13] S. Simons, The sequence spaces $\ell\left(p_{v}\right)$ and $m\left(p_{v}\right)$, Proc. London Math. Soc. 15(1965), 422-436.

[14] B. C. Tripathy, Matrix transformations between some classes of sequences, Jour. Math. Analysis \& Appl. 206(1997), 448-450.

[15] B. C. Tripathy, Matrix transformations between series and sequences, Bull. Malaysian Math. Soc. 22(1998), 17-20.

[16] B. C. Tripathy, Matrix maps on the power series convergent on the unit disc, Jour. Analysis 6(1998), 27-31.

[17] B. C. Tripathy and M. Sen, On generalized statistically convergent sequences, Ind. Jour. Pure \& Appl. Math. 32(2001), 1689-1694.

Mathematical Sciences Division, Institute of Advanced Study in Science and Technology, Paschim Boragaon, Garchuk, Guwahati- 781035, Assam, India.

E-mail: tripathybo@yahoo.com

Mathematical Sciences Division, Institute of Advanced Study in Science and Technology, Paschim Boragaon, Garchuk, Guwahati- 781035, Assam, India. 